Internat. J. Math. \& Math. Sci.

Vol. 24, No. 8 (2000) 511-517

S0161171200004361

(C) Hindawi Publishing Corp.

\title{
MULTIPLIERS OF BANACH VALUED WEIGHTED FUNCTION SPACES
}

\section{SERAP ÖZTOP}

(Received 24 June 1999 and in revised form 28 September 1999)

\begin{abstract}
We generalize Banach valued spaces to Banach valued weighted function spaces and study the multipliers space of these spaces. We also show the relationship between multipliers and tensor product of Banach valued weighted function spaces.
\end{abstract}

Keywords and phrases. Multiplier, weight function, convolution, Radon-Nikodym property, tensor product.

2000 Mathematics Subject Classification. Primary 43A22.

1. Introduction. We generalize Banach valued function spaces and its multipliers to the Banach valued weighted function spaces and its multipliers. We also get some elementary results.

Throughout we let $G$ be a locally compact abelian group with Haar measure, $X$ be a Banach space and $A$ be a commutative Banach algebra with identity of norm 1 . A weight, $\Psi$, is a measurable and strictly positive function on $G . L_{\Psi}^{p}(G, X)$ denote the space of equivalence classes of $X$-valued strongly measurable functions $f$ on $G$ such that $\Psi(t) f(t) \in L^{p}(G, X)$. The space $L_{\Psi}^{p}(G, X)$ is a Banach space normed by

$$
\|f\|_{L_{\Psi}^{p}(G, X)}=\left[\int_{G}\|f(t)\|_{X}^{p} \Psi(t)^{p} d t\right]^{1 / p}, \quad 1 \leq p<\infty,
$$

and its dual space of $L_{\Psi}^{p}(G, X)$ is $L_{\Psi^{-1}}^{p^{\prime}}\left(G, X^{*}\right)$, where $1 / p+1 / p^{\prime}=1$ if and only if $X^{*}$, the dual space of $X$, has the wide RNP (Radon-Nikodym Property) (see Lai [2] and Yoshikawa [6]). We assume that our weight function $\Psi$ will satisfy

$$
\Psi(s+t) \leq \Psi(s) \Psi(t), \quad s, t \in G,
$$

locally bounded and $w(x) \geq 1$, also $X^{*}$ and $X^{* *}$ have the wide RNP. It is known that every weight function is equivalent to a continuous weight function and we assume continuity (see Spector [5]). Let $C_{c}(G, X)$ denote the space of all $X$-valued continuous functions on $G$ with compact support and $C_{o}(G, X)$ the space of all $X$-valued continuous functions vanishing at infinity of $G$. For the weight function $\Psi$, let $C_{\Psi^{-1}}(G, X)$ denote the space of all functions $f$ such that $f \Psi^{-1} \in C_{o}(G, X)$ and define the norm as

$$
\|f\|_{C_{\infty, \Psi-1}(G, X)}=\sup _{t \in G} \frac{\|f(t)\|_{X}}{\Psi(t)} .
$$


A Banach space $X$ is said to be Banach $A$-module for a Banach algebra $A$ if $X$ is an $A$-module in the algebraic sense and satisfies

$$
\|a x\|_{X} \leq\|a\|\|x\|_{X}, \quad \text { for all } a \in A, x \in X .
$$

It is known that if $X$ is a Banach module, then $X^{*}$, the dual of $X$, is also a Banach module under the adjoint action of $A$. If $V$ and $W$ are Banach $A$-modules, then the $A$-module tensor product $V \otimes_{A} W$ is defined to be $\left(V \otimes_{\gamma} W\right) / K$, where $K$ is the closed linear subspace in the projective tensor product $V \otimes_{\gamma} W$ generated by the elements of the form

$$
a v \otimes \omega-v \otimes a \omega, \quad \text { for any } a \in A, v \in V, \omega \in W .
$$

(see Rieffel [4]), had shown that

$$
\operatorname{Hom}_{A}\left(V, W^{*}\right) \cong\left(V \otimes_{A} W\right)^{*}
$$

under which a linear functional $F$ on $V \otimes_{A} W$ corresponds to an operator $T \in \mathrm{Hom}_{A}$ $\left(V, W^{*}\right)$ such that

$$
(T v)(\omega)=F(v \otimes \omega), \quad \text { for all } v \in V, \omega \in W .
$$

The operator $T$, in fact, satisfies

$$
T(a v)=a T(v), \quad \text { for all } a \in A, v \in V,
$$

so is an $A$-module homomorphism which is a continuous linear transform from $V$ to $W^{*}$. We call this $A$-module homomorphism a multiplier (operator) from $V$ to $W^{*}$.

2. Some main properties of $L_{\Psi}^{p}(G, X)$. Let $G$ be a locally compact abelian group with Haar measure and $X$ be a Banach $A$-module. Then the $A$-valued functions acting on the $X$-valued functions are well defined. In the $X$-valued functions, we know that $L^{p}(G, X)$ is a Banach $L^{1}(G, A)$-module under convolution and $C_{c}(G, X)$ is dense in $L^{p}(G, X), 1 \leq p<\infty$ (Lai [2]). Now we will show that these properties are satisfied in the $X$-valued weighted functions.

Proposition 2.1. $C_{c}(G, X)$ is dense in $L_{\Psi}^{p}(G, X)$.

Proof. Let $f \in L_{\Psi}^{p}(G, X)$. Then $f \Psi \in L^{p}(G, X)$ given $\varepsilon>0$, there exists $f_{c} \in C_{c}$ $(G, X)$ such that

$$
\left\|f_{c}-f_{\Psi}\right\|_{L^{p}(G, X)}<\varepsilon
$$

Set $g_{c}=f_{c} / \Psi$. Then $g_{c} \in C_{c}(G, X)$ and $\left\|g_{c}-f\right\|_{L_{\Psi}^{p}(G, X)}<\varepsilon$. This completes the proof.

Proposition 2.2. Let $A$ be a commutative Banach algebra with identity of norm 1. $L_{\Psi}^{1}(G, A)$ is a commutative Banach algebra under convolution. 
Proof. Let $f, g \in L_{\Psi}^{1}(G, A)$. Then $f \Psi, g \Psi \in L^{1}(G, A)$. Since $L^{1}(G, A)$ is a commutative Banach algebra with the convolution we have

$$
\|f \Psi * g \Psi\|_{L^{1}(G, A)} \leq\|f\|_{L_{\Psi}^{1}(G, A)}\|g\|_{L_{\Psi}^{1}(G, A)} .
$$

Using the inequality (1.2) we can obtain easily

$$
\|f * g\|_{L_{\Psi}^{1}(G, A)} \leq\|f\|_{L_{\Psi}^{1}(G, A)}\|g\|_{L_{\Psi}^{1}(G, A)} .
$$

Proposition 2.3. Let $X$ be a Banach A-module. Then $L_{\Psi}^{p}(G, X)$ is a Banach $L_{\Psi}^{1}(G, A)$ module under convolution.

Proof. Let $f \in L_{\Psi}^{p}(G, X)$ and $g \in L_{\Psi}^{1}(G, A)$. Then $f \Psi \in L^{p}(G, X)$ and $g \Psi \in L_{\Psi}^{1}(G, A)$. Since $L^{p}(G, X)$ is a Banach $L^{1}(G, A)$ module with convolution,

$$
\|f \Psi * g \Psi\|_{L^{p}(G, X)} \leq\|f\|_{L_{\Psi}^{p}(G, X)}\|g\|_{L_{\Psi}^{p}(G, A)} .
$$

Using the inequality

$$
\|f * g\|_{L_{\Psi}^{p}(G, X)} \leq\|f \Psi * g \Psi\|_{L^{p}(G, X)},
$$

we obtain the module inequality

$$
\|f * g\|_{L_{\Psi}^{p}(G, X)} \leq\|f\|_{L_{\Psi}^{p}(G, X)}\|g\|_{L_{\Psi}^{1}(G, A)} .
$$

3. Multipliers from $L_{\Psi}^{p}(G, X)$ to $L_{\Psi-1}^{q^{\prime}}\left(G, X^{*}\right)$. Throughout we will assume that $\Psi$ is even weight function satisfying (1.2), $X$ is a Banach algebra, $1 / p+1 / q \geq 1,1<p<\infty$, and $1<q<\infty$.

Analogous to the scalar function case, we can obtain the following proposition.

Proposition 3.1. Let $f \in L_{\Psi}^{p}(G, X)$ and $g \in L_{\Psi}^{q}(G, X)$, where $1 / p+1 / q \geq 1$, $1 \leq p, q<\infty$. Then

$$
\|f * g\|_{L_{\Psi}^{r}(G, X)} \leq\|f\|_{L_{\Psi}^{p}(G, X)}\|g\|_{L_{\Psi}^{q}(G, X)},
$$

where $1 / r=1 / p+1 / q-1$. In fact, if $1 / p+1 / q=1$, then

$$
\|f * g\|_{C_{\infty, \Psi^{-1}}(G, X)} \leq\|f\|_{L_{\Psi}^{p}(G, X)}\|g\|_{L_{\Psi^{-1}}^{q}\left(G, X^{*}\right)} .
$$

Thus by Proposition 3.1 we can define a bilinear map, $b$, from $L_{\Psi}^{p}(G, X) x L_{\Psi}^{q}(G, X)$ into $L_{\Psi}^{r}(G, X)$ or $C_{\infty, \Psi^{-1}}(G, X)$ by

$$
b(f, g)=\tilde{f} * g, \quad \tilde{f}(x)=f(-x),
$$

and $\|b\| \leq 1$. Then $b$ lifts to a linear map, $B$, from $L_{\Psi}^{p}(G, X) \otimes_{\gamma} L_{\Psi}^{q}(G, X)$ into $L_{\Psi}^{r}(G, X)$ or $C_{\infty, \Psi^{-1}}(G, X)$ and $\|B\| \leq 1$ (see Bonsall-Duncan [1]). 
Definition 3.2. The range of $B$, with the quotient norm, will be denoted by $A_{\Psi}^{p, q}$ $(G, X)$. Thus $A_{\Psi}^{p, q}(G, X)$ is a Banach space of functions on $G$ which can be viewed as a linear submanifold in $L_{\Psi}^{r}(G, X)$ or $C_{\infty, \Psi-1}(G, X)$. In view of the fact that every element of $L_{\Psi}^{p}(G, X) \otimes_{\gamma} L_{\Psi}^{q}(G, X)$ has expansion of the form

$$
h=\sum_{i=1}^{\infty} f_{i} \otimes g_{i}, \quad f_{i} \in L_{\Psi}^{p}(G, X), g_{i} \in L_{\Psi}^{q}(G, X),
$$

where

$$
\sum_{i=1}^{\infty}\left\|f_{i}\right\|_{L_{\Psi}^{p}(G, X)}\left\|g_{i}\right\|_{L_{\Psi}^{q}(G, X)}<\infty,
$$

we see that $A_{\Psi}^{p, q}(G, X)$ consists of exactly those functions, $h$, on $G$ which have at least one expansion of the form

$$
h=\sum_{i=1}^{\infty} \tilde{f}_{i} * g_{i},
$$

where $f_{i} \in L_{\Psi}^{p}(G, X), g_{i} \in L_{\Psi}^{q}(G, X)$, and

$$
\sum_{i=1}^{\infty}\left\|f_{i}\right\|_{L_{\Psi}^{p}(G, X)}\left\|g_{i}\right\|_{L_{\Psi}^{q}(G, X)}<\infty,
$$

with the expansion converging in the norm of $L_{\Psi}^{r}(G, X)$ or $C_{\infty, \Psi-1}(G, X)$. As before, we let $K$ denote the closed subspace of $L_{\Psi}^{p}(G, X) \otimes_{L_{\Psi}^{1}(G, A)} L_{\Psi}^{q}(G, X)$ spanned by elements of the form $(\varphi * f) \otimes g-f \otimes(\tilde{\varphi} * g)$, where $\varphi \in L_{\Psi}^{1}(G, A), f \in L_{\Psi}^{p}(G, X)$, and $g \in L_{\Psi}^{q}(G, X)$.

Proposition 3.3. Let $1 / p+1 / q \geq 1$ and let $\phi \in C_{c}(G, X)$. Define $T_{\phi}$ by $T_{\phi}(f)=$ $f * \phi, f \in L_{\Psi}^{p}(G, X)$. Then $T_{\phi} \in \operatorname{Hom}_{L_{\Psi}^{1}(G, A)}\left(L_{\Psi}^{p}(G, X), L_{\Psi^{-1}}^{q^{\prime}}\left(G, X^{*}\right)\right)$. In fact

$$
\left\|T_{\phi}\right\| \leq\|\phi\|_{L_{\Psi-1}^{1}(G, A)}^{p / q^{\prime}}\|\phi\|_{L_{\Psi-1}^{p^{\prime}}}^{1-p / q^{\prime}}\left(G, X^{*}\right) .
$$

Proof. Let $f \in L_{\Psi}^{p}(G, X)$ and $g \in L_{\Psi}^{1}(G, A)$. It is easy to see that

$$
T_{\phi}(g * f)=g * T_{\phi}(f) .
$$

From the relations

$$
\begin{aligned}
\|f * \phi\|_{L_{\Psi-1}^{p}(G, X)} & \leq\|\phi\|_{L_{\Psi-1}^{1}(G, A)}\|f\|_{L_{\Psi}^{p}(G, X)}, \\
\|f * \phi\|_{C_{\infty, \Psi^{-1}}(G, X)} & \leq\|f\|_{L_{\Psi}^{p}(G, X)}\|\phi\|_{L_{\Psi^{-1}}^{p^{\prime}\left(G, X^{*}\right)}},
\end{aligned}
$$

we obtain

$$
\begin{aligned}
\left\|T_{\phi}(f)\right\|_{L_{\Psi^{-1}}^{q^{\prime}\left(G, X^{*}\right)}}^{q^{\prime}} & =\left\|(f * \phi) \Psi^{-1}\right\|_{L^{q^{\prime}\left(G, X^{*}\right)}}^{q^{\prime}} \\
& \leq\left\|(f * \phi) \Psi^{-1}\right\|_{C_{\infty}(G, X)}^{q^{\prime}-p}\left\|(f * \phi) \Psi^{-1}\right\|_{L^{p}(G, X)}^{p} \\
& \leq\left[\|\phi\|_{L_{\Psi^{-1}}^{p^{\prime}\left(G, X^{*}\right)}}^{q^{\prime}-p}\|\phi\|_{L_{\Psi-1}^{1}(G, X)}^{p}\right]\|f\|_{L_{\Psi}^{p}(G, X)}^{q^{\prime}},
\end{aligned}
$$


so that

$$
\left\|T_{\phi}(f)\right\|_{L_{\Psi^{-1}}^{q^{\prime}\left(G, X^{*}\right)}} \leq\|f\|_{L_{\Psi}^{p}(G, X)}\|\phi\|_{L_{\Psi^{-1}}^{p^{p^{\prime}}\left(G, Q^{*}\right)}}^{1-p / q^{\prime}}\|\phi\|_{L_{\Psi^{-1}}^{1}(G, A)}^{p / q^{\prime}}
$$

We finally obtain

$$
\left\|T_{\phi}\right\| \leq\|\phi\|_{L_{\Psi^{-1}}^{1}(G, A)}^{p / q^{\prime}}\|\phi\|_{L_{\Psi-1}^{p^{\prime}\left(G, X^{*}\right)}}^{1-p / q^{\prime}} .
$$

DEFINITION 3.4. We shall say that $\left\{L_{\Psi}^{p}(G, X), L_{\Psi^{-1}}^{q^{\prime}}\left(G, X^{*}\right)\right\}$ satisfies the approximation hypothesis if every element of $\operatorname{Hom}_{L_{\Psi}^{1}(G, A)}\left(L_{\Psi}^{p}(G, X), L_{\Psi^{-1}}^{q^{\prime}}\left(G, X^{*}\right)\right)$ can be approximated in the ultraweak operator topology by operators of the form $T_{\phi}, \phi \in C_{c}(G, X)$.

THEOREM 3.5. Let $1 / p+1 / q \geq 1$. Then the following statements are equivalent:

(i) $\left\{L_{\Psi}^{p}(G, X), L_{\Psi-1}^{q^{\prime}}\left(G, X^{*}\right)\right\}$ satisfies the approximation hypothesis.

(ii) The kernel of $B$ is $K$, so that $L_{\Psi}^{p}(G, X) \otimes_{L_{\Psi}^{1}(G, A)} L_{\Psi}^{q}(G, X) \cong A_{\Psi}^{p, q}(G, X)$.

Proof. Suppose now that $\left\{L_{\Psi}^{p}(G, X), L_{\Psi^{-1}}^{q^{\prime}}\left(G, X^{*}\right)\right\}$ satisfies the approximation hypothesis. It is easy to see that $K$ is always contained in the kernel of $B$ using the definition of $B$.

To show that the kernel of $B$ is contained in $K$ it suffices to show that $K^{\perp} \subset(\operatorname{Ker} B)^{\perp}$. Let $F \in K^{\perp}$ be given. Because of the isometric isomorphism

$$
K^{\perp} \cong\left[L_{\Psi}^{p}(G, X) \otimes_{L_{\Psi}^{1}(G, A)} L_{\Psi}^{q}(G, X)\right]^{*} \cong \operatorname{Hom}_{L_{\Psi}^{1}(G, A)}\left[L_{\Psi}^{p}(G, X), L_{\Psi^{-1}}^{q^{\prime}}\left(G, X^{*}\right)\right]
$$

there corresponds to $F$ a multiplier $T \in \operatorname{Hom}_{L_{\Psi}^{1}(G, A)}\left[L_{\Psi}^{p}(G, X), L_{\Psi^{-1}}^{q^{\prime}}\left(G, X^{*}\right)\right]$ such that

$$
\langle h, F\rangle=\sum_{i=1}^{\infty}\left\langle g_{i}, T f_{i}\right\rangle,
$$

where $h \in \operatorname{Ker} B, \sum_{i=i}^{\infty} f_{i} \otimes g_{i}$ and $\sum_{i=i}^{\infty}\left\|f_{i}\right\|_{L_{\Psi}^{p}(G, X)}\left\|g_{i}\right\|_{L_{\Psi}^{p}(G, X)}$. Moreover, since $\left\{L_{\Psi}^{p}(G, X)\right.$, $\left.L_{\Psi^{-1}}^{q^{\prime}}\left(G, X^{*}\right)\right\}$ satisfies the approximation hypothesis, there exists a $\left(T_{\phi_{j}}\right), \phi_{j} \in$ $C_{C}(G, X)$ such that

$$
\lim _{j} \sum_{i=1}^{\infty}\left\langle g_{i}, f_{i} * \phi_{j}\right\rangle=\sum_{i=1}^{\infty}\left\langle g_{i}, T f_{i}\right\rangle .
$$

Also it is easy to see that

$$
\sum_{i=1}^{\infty}\left\langle g_{i}, f_{i} * \phi_{j}\right\rangle=\sum_{i=1}^{\infty}\left\langle\tilde{f}_{i} * g_{i}, \phi_{j}\right\rangle .
$$

Using the Hölder inequality, we obtain

$$
\begin{aligned}
\left|\sum_{i=1}^{\infty}\left\langle g_{i}, f_{i} * \phi_{j}\right\rangle\right| & =\left|\sum_{i=1}^{\infty}\left\langle\tilde{f}_{i} * g_{i}, \phi_{j}\right\rangle\right| \\
& \leq\left\|\sum_{i=1}^{\infty} \tilde{f}_{i} * g_{i}\right\|_{L_{\Psi}^{r}(G, X)}\left\|\Phi_{j}\right\|_{L_{\Psi^{\prime}-1}^{r^{\prime}}\left(G, X^{*}\right)}=0
\end{aligned}
$$


hence if we combine (3.16) and (3.18), we find

$$
\langle h, F\rangle=\sum_{i=1}^{\infty}\left\langle g_{i}, T f_{i}\right\rangle=0 .
$$

Therefore, $\langle h, F\rangle=0$ for all $h \in \operatorname{Ker} B$. That means $F \in(\operatorname{Ker} B)^{\perp}$. This proves $L_{\Psi}^{p}(G, X) \otimes_{L_{\Psi}^{1}(G, A)} L_{\Psi}^{q}(G, X) \cong A_{\Psi}^{p, q}(G, X)$.

Suppose conversely that $\operatorname{Ker} B=K$. We will show that the set $N=\left\{T_{\phi} \mid \phi \in C_{C}(G, X)\right\}$ is dense in $\operatorname{Hom}_{L_{\Psi}^{1}(G, A)}\left(L_{\Psi}^{p}(G, X), L_{\Psi^{-1}}^{q^{\prime}}\left(G, X^{*}\right)\right)$ in the ultraweak operator topology. Because of the isometric isomorphism

$$
\operatorname{Hom}_{L_{\Psi}^{1}(G, A)}\left(L_{\Psi}^{p}(G, X), L_{\Psi^{-1}}^{q^{\prime}}\left(G, X^{*}\right)\right) \cong\left[L_{\Psi}^{p}(G, X) \otimes_{L_{\Psi}^{1}(G, A)} L_{\Psi}^{q}(G, X)\right]^{*},
$$

it is sufficient to show that the corresponding functionals are dense in $\left[L_{\Psi}^{p}(G, X)\right.$ $\left.\otimes_{L_{\Psi}^{1}(G, A)} L_{\Psi}^{q}(G, X)\right]^{*}$ in the weak*-topology. Let $M$ be the set of the linear functionals which corresponds to the operators in $\left[L_{\Psi}^{p}(G, X) \otimes_{L_{\Psi}^{1}(G, A)} L_{\Psi}^{q}(G, X)\right]^{*}$. To show this it is sufficient to denote that $M^{\perp}=\{0\}=K$. Using the $\operatorname{Ker} B=K$, it suffices to show $M^{\perp}=\operatorname{Ker} B$. Since

$$
\left[L_{\Psi}^{p}(G, X) \otimes_{L_{\Psi}^{1}(G, A)} L_{\Psi}^{q}(G, X)\right]^{*} \cong(\operatorname{Ker} B)^{\perp}
$$

then $\langle h, F\rangle=0$ for all $h \in \operatorname{Ker} B$ and $F \in M$. Thus $h \in M^{\perp}$. That means $\operatorname{Ker} B \subset M^{\perp}$. Conversely, if we use (3.17) and (3.19) we obtain that

$$
\left\langle\sum_{i=1}^{\infty} \tilde{f}_{i} * g_{i}, \phi\right\rangle=\langle h, F\rangle=0 .
$$

Since $\phi \in C_{c}(G, X)$ and hence can be viewed as an element of $L_{\Psi^{-1}}^{r}\left(G, X^{*}\right)$ and $\tilde{f}_{i} * g_{i} \in$ $L_{\Psi}^{r}(G, X)$ this implies that $\sum_{i=1}^{\infty} \tilde{f}_{i} * g_{i}=0$. Therefore $M^{\perp} \subset \operatorname{Ker} B$. Thus $M^{\perp}=\operatorname{Ker} B$. This proves the assertion.

COROLlary 3.6. Let $1 / p+1 / q \geq 1,1 / p+1 / q-1=1 / r$, and $1 / q+1 / q^{\prime}=1$. If $\left\{L_{\Psi}^{p}(G, X), L_{\Psi^{-1}}^{q^{\prime}}\left(G, X^{*}\right)\right\}$ satisfies the approximation hypothesis, then we have the identification

$$
\operatorname{Hom}_{L_{\Psi}^{1}(G, A)}\left[L_{\Psi}^{p}(G, X), L_{\Psi^{-1}}^{q^{\prime}}\left(G, X^{*}\right)\right] \cong\left[A_{\Psi}^{p, q}(G, X)\right]^{*}
$$

REMARK 3.7. For particular cases of Theorem 3.5, if weight function $\Psi=1$ then one can obtain the space of multipliers from $L^{p}(G, X)$ to the space $L^{q^{\prime}}\left(G, X^{*}\right)$.

If $\Psi=1, X=K(K=\mathbb{R}$ or $\mathbb{C})$ the space of multipliers from $L^{p}(G)$ to $L^{q^{\prime}}(G), G$ is a locally compact group, was studied in [4].

If $X=K(K=\mathbb{R}$ or $\mathbb{C})$ the space of multipliers from $L_{\Psi}^{p}(G)$ to $L_{\Psi^{-1}}^{q^{\prime}}(G)$ was studied (for $\Psi$ is a Beurling weight function and $G$ is unimodular group) in [3].

ACKNOwLedgement. I would like to thank H. C. Lai for his helpful suggestions. 


\section{REFERENCES}

[1] F. F. Bonsall and J. Duncan, Complete Normed Algebras, Ergebnisse der Mathematik und ihrer Grenzgebiete, no. 80, Springer-Verlag, Berlin, Heidlberg, New York, 1973. MR 54\#11013. Zbl 271.46039.

[2] H. C. Lai, Multipliers of Banach valued function spaces, J. Austral. Math. Soc. Ser. A 39 (1985), no. 1, 51-62. MR 86h:46060. Zbl 579.43006.

[3] S. Öztop and A. T. Gürkanli, Multipliers and tensor products of weighted $L^{p}$-spaces, preprint, 2000.

[4] M. A. Rieffel, Multipliers and tensor products of $L^{p}$-spaces of locally compact groups, Studia Math. 33 (1969), 71-82. MR 39\#6078. Zbl 177.41702.

[5] R. Spector, Sur la structure locale des groupes abéliens localement compacts, Bull. Soc. Math. France Suppl. Mém. 24 (1970), 94. MR 44\#729. Zbl 215.18603.

[6] A. Yoshikawa, Sur la théorie d'espaces d'interpolation-les espaces de moyenne de plusieurs espaces de Banach, J. Fac. Sci. Univ. Tokyo Sect. I 16 (1969), 407-468 (1970). MR 58\#30339. Zbl 197.09901.

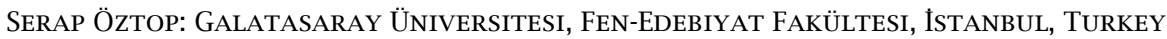

E-mail address: serapoztop@hotmai 1.com 


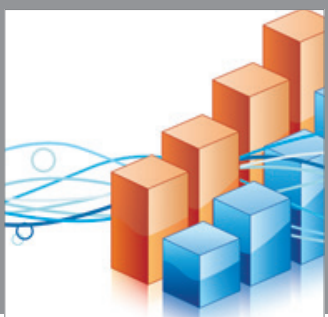

Advances in

Operations Research

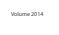

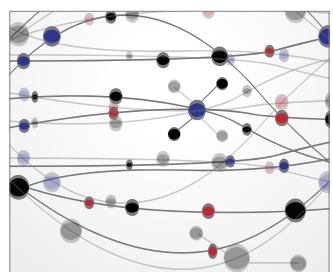

\section{The Scientific} World Journal
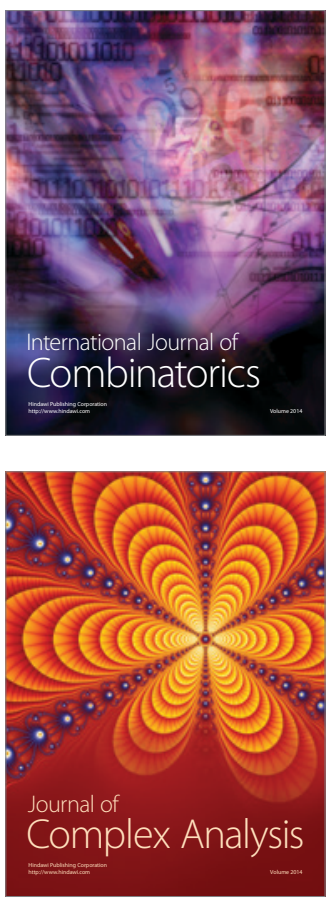

International Journal of

Mathematics and

Mathematical

Sciences
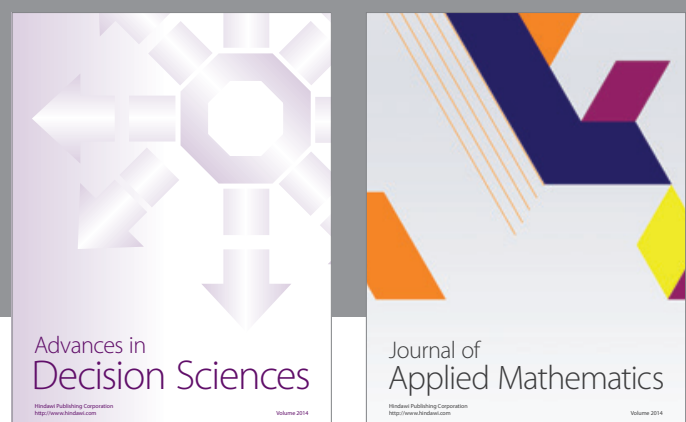

Journal of

Applied Mathematics
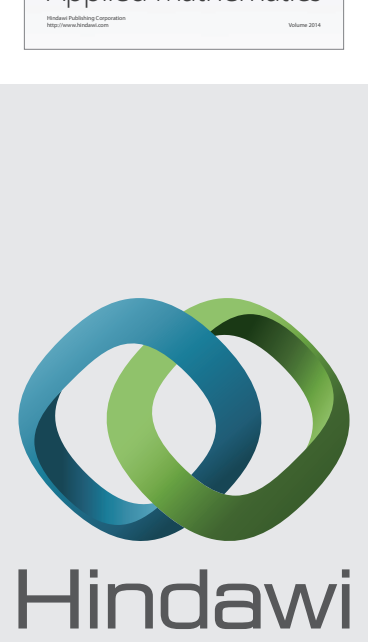

Submit your manuscripts at http://www.hindawi.com
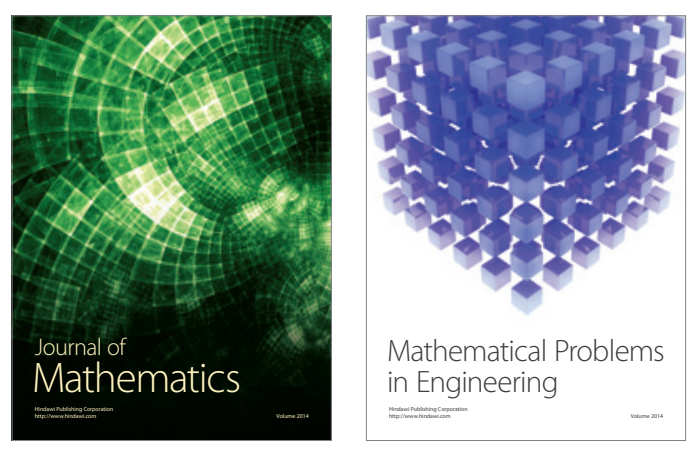

Mathematical Problems in Engineering
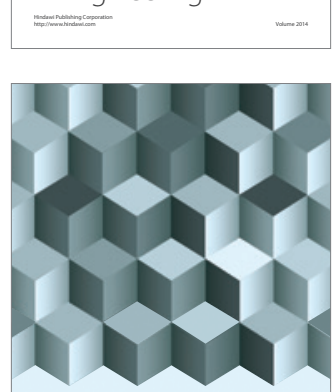

Journal of

Function Spaces
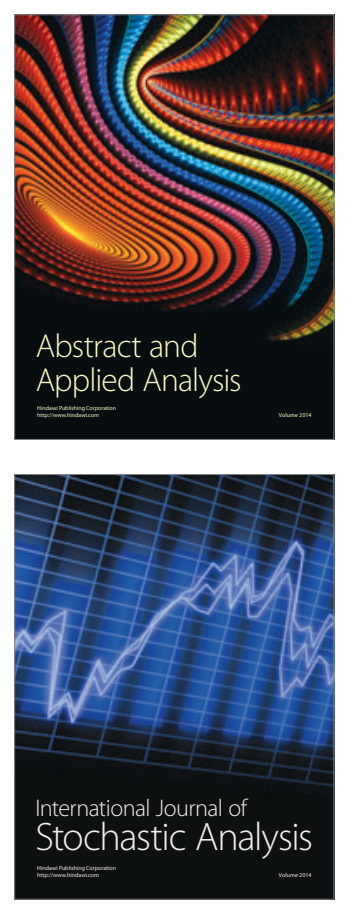

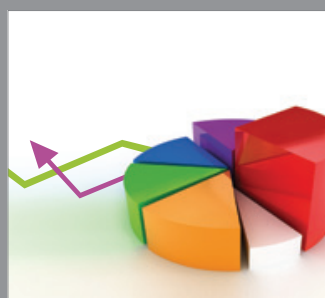

ournal of

Probability and Statistics

Promensencen
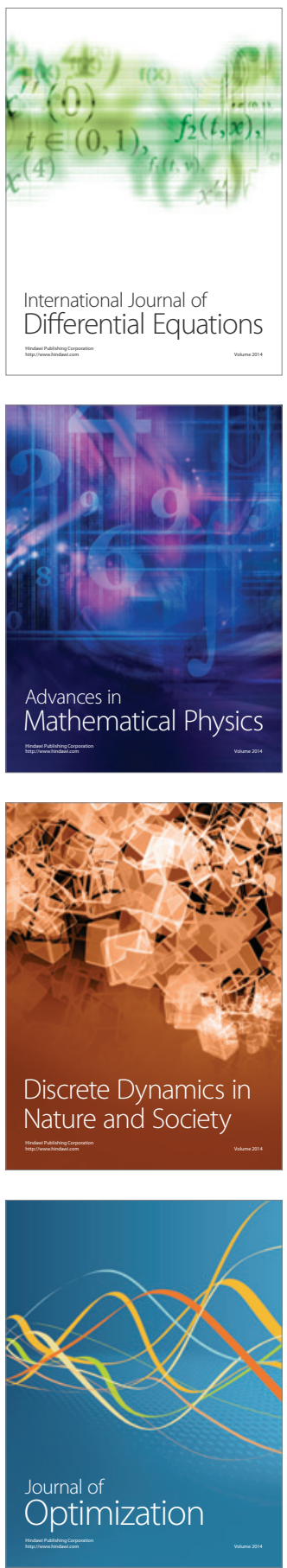